\title{
Erratum: The light-fermion contribution to the exact Higgs-gluon form factor in QCD
}

\author{
Robert V. Harlander, ${ }^{a}$ Mario Prausa ${ }^{b}$ and Johann Usovitsch ${ }^{c}$ \\ ${ }^{a}$ Institute for Theoretical Particle Physics and Cosmology, \\ RWTH Aachen University, Aachen D-52056, Germany \\ ${ }^{b}$ Physikalisches Institut, Albert-Ludwigs-Universität, \\ Freiburg D-79104, Germany \\ ${ }^{c}$ Trinity College Dublin, School of Mathematics, \\ Dublin 2, Ireland \\ E-mail: harlander@physik.rwth-aachen.de, \\ mario.prausa@physik.uni-freiburg.de, usovitsj@maths.tcd.ie
}

ERRATUM TO: JHEP10(2019)148

ARXIV EPRINT: 1907.06957 


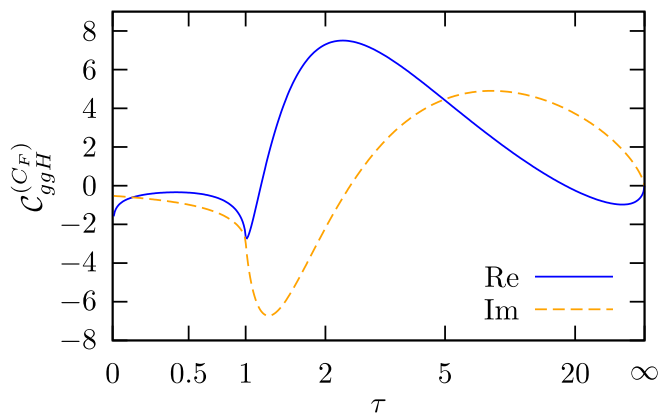

(a)

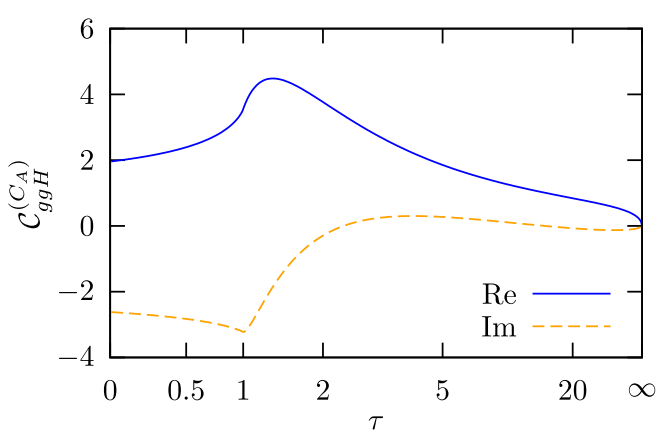

(b)

Figure 1. Contributions to $\tilde{C}_{g g H}^{(2)}$ separated by their color factors. The quark mass is renormalized in the on-shell scheme and the renormalization scale is set to $\mu^{2}=M_{\mathrm{H}}^{2}$.

As pointed out in ref. [1], the $H_{g}$-term of eq. $(3.7 \mathrm{~b})$ in the original paper is missing a renormalization scale-dependent prefactor and should read

$$
\begin{aligned}
I_{g}^{(2)}= & -\frac{1}{2} I_{g}^{(1)}(\epsilon)\left(I_{g}^{(1)}(\epsilon)+\frac{\beta_{0}}{\epsilon}\right)+\frac{e^{-\epsilon \gamma_{E}} \Gamma(1-2 \epsilon)}{\Gamma(1-\epsilon)}\left(\frac{\beta_{0}}{\epsilon}+K\right) I_{g}^{(1)}(2 \epsilon) \\
& +\left(-\frac{\mu^{2}}{M_{\mathrm{H}}^{2}}\right)^{2 \epsilon} \frac{e^{\epsilon \gamma_{E}}}{\Gamma(1-\epsilon)} \frac{H_{g}}{2 \epsilon} .
\end{aligned}
$$

This leads to additional contributions to eq. (3.9) of the original publication, which are proportional to $\ln \left(-\mu^{2} / M_{\mathrm{H}}^{2}\right)=L_{\mu}+2 H_{1}+H_{0}$. The corrected results read

$$
\begin{aligned}
& \mathcal{C}_{g g H, \text { corr. }}^{\left(C_{F}\right)}=\mathcal{C}_{g g H}^{\left(C_{F}\right)}-\frac{1}{4} \ln \left(-\frac{\mu^{2}}{M_{\mathrm{H}}^{2}}\right) \frac{C_{g g H}^{(0)}}{T_{F}}, \\
& \mathcal{C}_{g g H, \text { corr. }}^{\left(C_{A}\right)}=\mathcal{C}_{g g H}^{\left(C_{A}\right)}+\left(\frac{\pi^{2}}{144}+\frac{29}{54}\right) \ln \left(-\frac{\mu^{2}}{M_{\mathrm{H}}^{2}}\right) \frac{C_{g g H}^{(0)}}{T_{F}}, \\
& \tilde{C}_{g g H, \text { corr. }}^{(2,2)}=\tilde{C}_{g g H}^{(2,2)}-\frac{5}{27} \ln \left(-\frac{\mu^{2}}{M_{\mathrm{H}}^{2}}\right) T_{F}^{2} C_{g g H}^{(0)},
\end{aligned}
$$

where $C_{g g H}^{(0)}, \mathcal{C}_{g g H}^{\left(C_{F}\right)}, \mathcal{C}_{g g H}^{\left(C_{A}\right)}$ and $\tilde{C}_{g g H}^{(2,2)}$ are given by eq. (3.5), eq. (B.4), eq. (B.5) and eq. (B.9) in the original paper, respectively. Corrected plots for figure 3 of the original publication are shown in figure 1 (Note that figure 4 remains unchanged due to the scale choice $\mu^{2}=-M_{\mathrm{H}}^{2}$ ). An updated version of the file ggh-aah-nl.m containing the results in electronic form is attached to this erratum as supplementary material.

Open Access. This article is distributed under the terms of the Creative Commons Attribution License (CC-BY 4.0), which permits any use, distribution and reproduction in any medium, provided the original author(s) and source are credited. 


\section{References}

[1] M.L. Czakon and M. Niggetiedt, Exact quark-mass dependence of the Higgs-gluon form factor at three loops in QCD, JHEP 05 (2020) 149 [arXiv:2001.03008] [INSPIRE]. 